\title{
BIFURCATION SET AND LIMIT CYCLES FORMING COMPOUND EYES IN A PERTURBED HAMILTONIAN SYSTEM
}

\author{
JIBIN LI AND ZHENRONG LIU
}

\begin{abstract}
In this paper we consider a class of perturbation of a Hamiltonian cubic system with 9 finite critical points. Using detection functions, we present explicit formulas for the global and local bifurcations of the flow. We exhibit various patterns of cornpound eyes of limit cycles. These results are concerned with the weakened Hibbert's 16 th problem posed by V.I. Arnold in 1977 .
\end{abstract}

\section{Introduction}

The weakened Hilbert 16th problem, posed by V.I. Arnold in 1977 [1], is to determine the number of limit cycles that can be generated from a polynomial Hamiltonian system of degree $n-1$ with perturbed terms of a polynomial of degree $m+1$. The separatrixes and relative positions of the limit cycles for the Hamiltonian system with perturbations play an important role [2]. For a polynomial differential system of degree $n$, the results of [3] imply that, in order to get more limit cycles and various patterns of their distribution, one cfficient method is to perturb a Hamiltonian system with symmetry which has the maximal number of centers. Thus, to study the weakened Hilbert 16th problem, we should first investigate the property of unperturbed Hamiltonian systems, i.e., determine the global property of the family of planar algebraic curves. Then, by using proper perturbation techniques, we can obtain the global information of the perturbed non-integrable system.

Only two particular examples were given in the paper [3]. In this paper we discuss the following system:

$$
\begin{aligned}
& \frac{d x}{d t}=y\left(1+x^{2}-a y^{2}\right)+\varepsilon x\left(m x^{2}+n y^{2}-\lambda\right), \\
& \frac{d y}{d t}=-x\left(1-c x^{2}+y^{2}\right)+\varepsilon y\left(m x^{2}+n y^{2}-\lambda\right),
\end{aligned}
$$

where $a>c>0, a c>1,0<\varepsilon<<1, m, n, \lambda$ are parameters. Our object is to reveal the bifurcation set in the 5 -parameter space. Since the vector field defined 
by $(1.1)_{\varepsilon=0}$ is invariant under the rotation over $\pi$, the phase portrait of $(1.1)_{\varepsilon}$ has a high degree of symmetry. By bifurcating limit cycles from homoclinic and heteroclinic orbits and centers, we obtain many interesting distributions of limit cycles which form various patterns of compound eyes.

It is well known that a point is defined to belong to the bifurcation set if, in any neighbourhood in the parameter values, there exist at least two topologically distinct phase portraits. By computing detection functions [5] [6], we can give a description of the bifurcation set in the five-parameter space of (1.1 $)_{\varepsilon}$. For the fixed pair of $a, c$, the half parameter plane $(n, m)$ with $m \geq 0$ can be partitioned into 19 angle regions. Hence, various possible phase portraits of $(1.1)_{\varepsilon}$ can be found. Especially, for a complex polynomial system, Il'jasenko [7] has proved that with applications to real cases, the cubic system has 5 limit cycles with disjoint interiors. This paper shows that there exist a large region of parameters such that the Il'jasenko distribution of limit cycles can be realized by $(1.1)_{\varepsilon}$.

The first author has been supported by the C. C. Wu Cultural \& Education Foundation fund Ltd. in Hong Kong. Moreover, he is indebted to Jack K. Hale and Shui-Nee Chow for helpful discussions.

\section{Analysis of the unperturbed system}

Consider the system

$$
\frac{d x}{d t}=y\left(\mathrm{I}+x^{2}-a y^{2}\right), \quad \frac{d y}{d t}=-x\left(1-c x^{2}+y^{2}\right) .
$$

The system (2.1) has 9 finite singular points, among them, $0(0,0)$, $A_{1}^{0}\left(\sqrt{\frac{1+a}{a c-1}}, \sqrt{\frac{1+c}{a c-1}}\right)$ and $A_{2}^{0}, \ldots, A_{4}^{0}$ (sce Fig. 2.1) are centers; $S_{1}^{0}(1 / \sqrt{c}, 0)$, $S_{2}^{0}(-1 / \sqrt{c}, 0), S_{3}^{0}(0,1 / \sqrt{a})$ and $S_{4}^{0}(0,-1 / \sqrt{a})$ are hyperbolic saddle points. For $0<\varepsilon<<1,(1.1)_{\epsilon}$ also has 9 critical points, 0 and $A_{i}, S_{i}(i=1, \ldots, 4)$, which take respectively slight displacements from $A_{i}^{0}$ and $S_{i}^{0}$.

The first integral of (2.I) is given by

$$
H(x, y)=-\left(c x^{4}+a y^{4}\right)+2 x^{2} y^{2}+2\left(x^{2}+y^{2}\right)=h .
$$

Its polar coordinate form is

$$
\begin{gathered}
H(r, \theta)=-r^{4}\left(c \cos ^{4} \theta+a \sin ^{4} \theta-2 \cos ^{2} \theta \sin ^{2} \theta\right)+2 r^{2} \\
\stackrel{\text { def }}{=}-r^{4} u(\theta)+2 r^{2}=h .
\end{gathered}
$$

If we let $x=r \cos \theta, y=r \sin \theta$, then (2.1) becomes

$$
\frac{d r}{d t}=r^{3} u^{\prime}(\theta), \quad \frac{d \theta}{d t}=-\left(1-r^{2} u(\theta)\right) .
$$




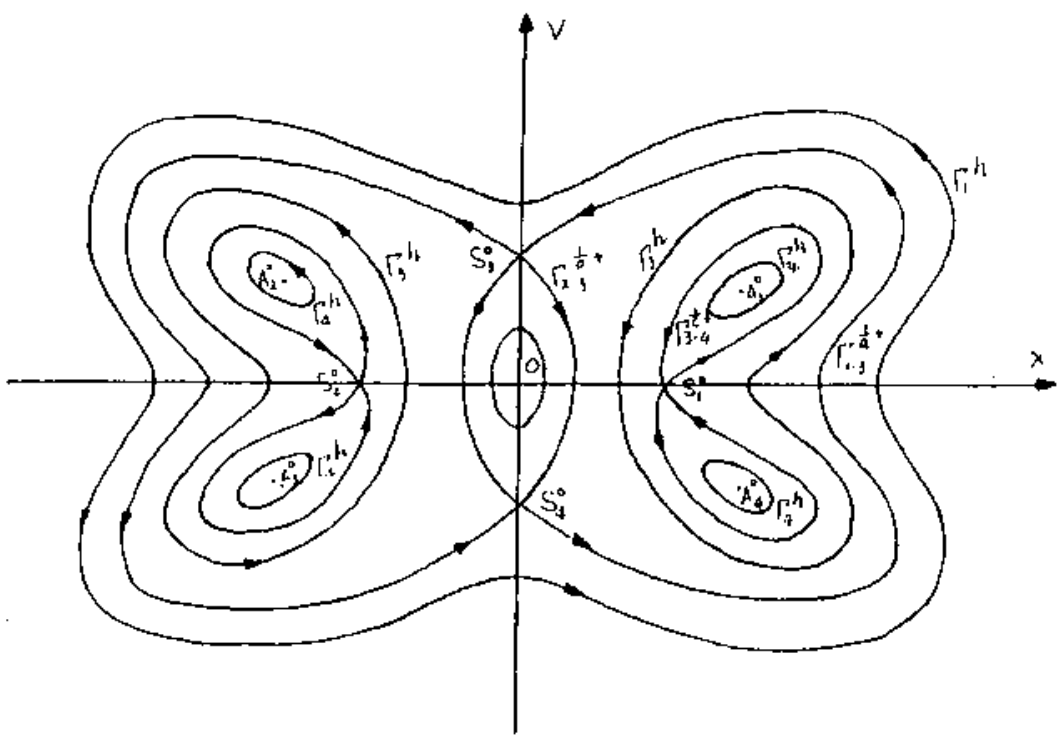

Fig. 2.1 The phase portrait of unperturbed system (2.1).

From (2.3), we have

$$
r^{2}=r_{ \pm}^{2}(\theta, h)=\frac{1 \pm \sqrt{1-h u(\theta)}}{u(\theta)} \stackrel{\text { def }}{=} \frac{1 \pm \sqrt{v(\theta, h)}}{u(\theta)}
$$

Using (2.3) - (2.5), it follows that

(2.6)

$\frac{d \theta}{d t}= \pm \sqrt{v(\theta, h)}= \pm \frac{1}{2}\left[-(a+c+2) h \cos ^{2} 2 \theta+2 h(a-c) \cos 2 \theta+4-h(a+c-2)\right]^{1 / 2}$.

With $h$ varying, the curves defined by (2.2) can be divided into the following types:

(i) $\left\{\Gamma_{1}^{h}\right\}:-\infty<h<-1 / a$. This corresponds to a family of closed orbits which enclose all 9 finite singular points. Let $s n u, c n u$, dnu be the Jacobian elliptic functions with modulus $k$. If we denote by

$$
\mu_{h}(t)=\left[\omega_{1} \sqrt{\rho \bar{\rho}}\left(k_{1}^{2} c n^{2} \omega_{1} t s n^{2} \omega_{1} t-d n^{2} \omega_{1} t\right)-\rho \bar{\rho} c n^{2} \omega_{1} t-d n^{2} \omega_{1} t s n^{2} \omega_{1} t\right]^{1 / 2}
$$

where $k_{1}=\left[\frac{-(\rho-\bar{\rho})^{2}}{4 \rho \bar{\rho}}\right], \omega_{1}=2 \sqrt{(1-a h) \rho \bar{\rho}}$

$$
\rho=\frac{-(h+1)+i\left((a c-1) h^{2}-(a+c+2) h\right)^{1 / 2}}{1-a h}
$$


Then the orbit of $\left\{\Gamma_{1}^{h}\right\}$ has the parametric representation

$$
x_{1}(t)=\frac{\sqrt{-h} d n \omega_{1} t \operatorname{snn} \omega_{1} t}{\mu_{h}(t)}, \quad y_{1}(t)=\frac{\sqrt{-h \rho \bar{\rho}} c n \omega_{1} t}{\mu_{h}(t)},
$$

where $-\infty<h<0$.

(ii) $\left\{\Gamma_{2}^{h}\right\}: 0<h<\frac{1}{a}$. This corresponds to a family of closed curves surrounding the origin $0(0,0)$, which has the parametric representation

$$
x_{2}(t)=\frac{\sqrt{h} d n \omega_{1} t s n \omega_{1} t}{\mu_{h}(t)}, \quad y_{2}(t)=\frac{\sqrt{h \rho \bar{\rho}} c n \omega_{1} t}{\mu_{h}(t)} .
$$

(iii) When $h=1 / a$, there are 4 heteroclinic orbits connecting two critical points $S_{3}^{0}$ and $S_{4}^{0}$. If we let

$$
\mu_{1 / a}^{ \pm}(t)=\left[a\left(A_{1}^{2} \operatorname{sh}^{2} 2 \beta_{1} t \pm 2 A_{1} \beta_{1} \operatorname{ch} 2 \beta_{1} t+1\right]^{1 / 2}\right.
$$

where $A_{1}^{2}=(a-c) / 2(a+1), \beta_{1}^{2}=2(a+1) / a$, then the heteroclinic orbits $\Gamma_{2,3}^{1 / a+}$ and $\Gamma_{1,3}^{1 / a+}$ have respectively parametric representation:

$$
\begin{array}{ll}
\Gamma_{2,3}^{1 / a+}: x_{2,3}(t)=\frac{1}{\mu_{1 / a}^{+}(t)}, & y_{2,3}(t)=\frac{-A_{1} \operatorname{sh} 2 \beta_{1} t}{\mu_{1 / a}^{+}(t)}, \\
\Gamma_{1,3}^{1 / a+}: x_{1,3}(t)=\frac{1}{\mu_{1 / a}^{-}(t)}, & y_{1,3}(t)=\frac{A_{1} s h 2 \beta_{1} t}{\mu_{1 / a}^{-}(t)}
\end{array}
$$

(iv) $\left\{\Gamma_{3}^{h \pm}\right\}: \frac{1}{a}<h<\frac{1}{c}$. These are two families of closed orbits surrounding respectively three singular points $A_{1}^{0}, S_{1}^{0}, A_{4}^{0}$ and $A_{2}^{0}, S_{2}^{0}, S_{3}^{0}$. The orbit of $\left\{\Gamma_{3}^{h+}\right\}$ has the parametric representation:

$$
\begin{aligned}
& x_{3}(t)=\sqrt{h}\left[1+2 \alpha \omega_{3} d n 2 \omega_{3} t \operatorname{sn} 2 \omega_{3} t\right]^{-1 / 2}, \\
& y_{3}(t)=\sqrt{h}\left[1+2 \alpha \omega_{3} d n 2 \omega_{3} t \operatorname{sn} 2 \omega_{3} t\right]^{-1 / 2} \alpha \operatorname{cn} 2 \omega_{3} t
\end{aligned}
$$

where $\omega_{3}=\sqrt{2} \Delta^{1 / 4}, \Delta=h(-a(a c-1) h+(a+c+2)]$, the modulo $k_{3}=$ $\alpha / \sqrt{\alpha^{2}+\beta^{2}}, \alpha^{2}=\frac{(l+h)+\sqrt{\Delta}}{2 h-1}, \beta^{2}=\frac{\sqrt{\Delta}-(1+h)}{a h-1}$.

(v) When $h=\frac{1}{6}$, there are four homoclinic orbits $\Gamma_{3,4}^{1 / c \pm}$ surrounding respectively the centers $A_{i}^{0}(i=1-4)$ and connecting respectively the saddle points $S_{1}^{0}$ and $S_{2}^{0}$. One of homoclinic orbits has the parametric representation:

$$
\begin{aligned}
\Gamma_{3,4}^{1 / c t}: x_{3,4}(t) & =\frac{\operatorname{ch} 2 \beta_{2} t}{\sqrt{c}\left[\operatorname{sh}^{2} 2 \beta_{2} t+2 A_{2} \beta_{2} \operatorname{sh} 2 \beta_{2} t+\left(1-A_{2}^{2}\right)\right]^{1 / 2}}, \\
y_{3,4}(t) & =\frac{1}{\sqrt{c}\left[\operatorname{sh}^{2} 2 \beta_{2} t+2 A_{2} \beta_{2} \operatorname{sh} 2 \beta_{2} t+\left(1-A_{2}^{2}\right)\right]^{1 / 2}},
\end{aligned}
$$


where $\beta_{2}^{2}=2(1+c) / c, A_{2}^{2}=2(1+c) /(a-c)$.

(vi) $\left\{\Gamma_{4}^{h}\right\}: \frac{1}{c}<h<\frac{a+c+2}{a c-1}$. This is composed of four families of closed orbits surrounding respectively one critical point $A_{i}^{0}(i=1-4)$. If we write

$$
\nu_{h}(t)=\left[1+2 \alpha \omega_{4} k_{4}^{2} \operatorname{sn} 2 \omega_{4} t \operatorname{cn} 2 \omega_{4} t+\alpha^{2} d n^{2} 2 \omega_{4} t\right]^{1 / 2},
$$

where $\omega_{4}=[(1+h)+\sqrt{\Delta}]^{1 / 2}, k_{4}^{2}=\left(\alpha^{2}-\beta^{2}\right) / \alpha^{2}, \alpha, \beta$ are the same as in (iv), then one family of $\left\{\Gamma_{4}^{h}\right\}$ has the parametric representation:

$$
x_{4}(t)=\frac{\sqrt{h}}{\nu_{h}(t)}, \quad y_{4}(t)=\frac{\sqrt{h} \alpha d n 2 \omega_{4} t}{\nu_{h}(t)} .
$$

Note that as $h$ increasing, the curve $\Gamma_{2}^{h}$ extends outside, the other curves constrict inside.

\section{Detection functions and bifurcation parameter of the perturbed system}

In the paper [5], we have considered the perturbed Hamiltonian system

$$
\begin{aligned}
& \frac{d x}{d t}=\frac{\partial H}{\partial y}-\varepsilon x(p(x, y)-\lambda), \\
& \frac{d y}{d t}=-\frac{\partial H}{\partial x}-\varepsilon y(q(x, y)-\lambda),
\end{aligned}
$$

where $H(x, y)=h$ is a first integral of $(3.0)_{\varepsilon=0}$. Assume $(\xi, \eta)$ is a critical point of $(3.0)_{\varepsilon=0}$, and there exists a family $\left\{\Gamma^{h}\right\}$ of closed orbits surrounding $(\xi, \eta)$ when $0<h<\hat{h}$. We call the function

$$
\lambda(h)=\int_{\Gamma_{h}} \int f(x, y) d x d y / 2 \int_{\Gamma_{h}} \int d x d y \quad(0<h<\hat{h})
$$

a detection function, where $f(x, y)=p+q+x \frac{\partial p}{\partial x}+y \frac{\partial q}{\partial y}$. Obviously, if (3.0) is a polynomial system, then $\lambda(h)$ is the ratio of two Abelian integrals [8]. By using $\lambda(h)$, we can determine the existence and stability of limit cycles created by $\left\{\Gamma^{h}\right\}$.

We know the following conclusions from [5] and [6]:

(i) The parametric value of the Hopf bifurcation created by the critical point $(\xi, \eta)$ is $\lambda(0)=\frac{1}{2} f(\xi, \eta)$.

(ii) If $(3.0)_{\varepsilon}$ has a homoclinic orbit $\Gamma^{\hat{h}}$ at $h=\hat{h}$, which connects a hyperbolic critical point $(\alpha, \beta)$, then the parametric value of the homoclinic bifurcation is $\lambda(\hat{h})=\lim _{h \rightarrow \hat{h}} \lambda(h)$.

(iii) The sign of $\lambda^{\prime}(\hat{h})$ is determined by the sign of saddle value of $(\alpha, \beta)$. 
We now consider the perturbed system $(1.1)_{\varepsilon}$. By using the method of [3] - [5], corresponding to 4 families of closed orbits $\left\{\Gamma_{i}^{h}\right\}(i=1-4)$, we define 4 detection functions as follows:

$$
\begin{aligned}
\lambda_{1}(h) & =\frac{\psi_{1}(h)}{\phi_{1}(h)}=\int_{0}^{\pi / 2} r_{+}^{4}(\vartheta, h) g(\vartheta) d \vartheta / \int_{0}^{\pi / 2} r_{+}^{2}(\vartheta, h) d \vartheta \\
& =\int_{0}^{\pi / 2}\left[\frac{1+\sqrt{v(\vartheta, h)}}{u(\vartheta)}\right]^{2} g(\vartheta) d \vartheta / \int_{0}^{\pi / 2}\left[\frac{1+\sqrt{v(\vartheta, h)}}{u(\vartheta)}\right] d \vartheta
\end{aligned}
$$

$$
\begin{aligned}
\lambda_{2}(h) & =\frac{\psi_{2}(h)}{\phi_{2}(\bar{h})}=\int_{0}^{\pi / 2} r_{-}^{4}(\vartheta, h) g(\vartheta) d \vartheta / \int_{0}^{\pi / 2} r_{-}^{2}(\vartheta, h) d \vartheta \\
& =\int_{0}^{\pi / 2}\left[\frac{1-\sqrt{v(\vartheta, h)}}{u(\vartheta)}\right]^{2} g(\vartheta) d \vartheta / \int_{0}^{\pi / 2}\left[\frac{1-\sqrt{v(\vartheta, h)}}{u(\vartheta)}\right] d \vartheta
\end{aligned}
$$

$$
\begin{aligned}
\lambda_{3}(h) & =\frac{\psi_{3}(h)}{\phi_{3}(h)}=\int_{0}^{\dot{\vartheta}(h)}\left[r_{+}^{4}(\vartheta)-r_{-}^{4}(\vartheta)\right] g(\vartheta) d \vartheta / \int_{0}^{\dot{\vartheta}(h)}\left[r_{+}^{2}(\vartheta)-r_{-}^{2}(\vartheta)\right] d \vartheta \\
& =\int_{0}^{\vartheta(h)} \frac{2 \sqrt{v(\vartheta, h)}}{u^{2}(\vartheta)} g(\vartheta) d \vartheta / \int_{0}^{\dot{\vartheta}(h)} \frac{\sqrt{v(\vartheta, h)}}{u(\vartheta)} d \vartheta
\end{aligned}
$$

$$
\begin{array}{r}
\lambda_{4}(h)=\frac{\psi_{4}(h)}{\phi_{4}(h)}=\int_{\vartheta_{2}(h)}^{\vartheta_{3}(h)} \frac{2 \sqrt{v(\vartheta, h)}}{u^{2}(\vartheta)} g(\vartheta) d \vartheta / \int_{\vartheta_{2}(h)}^{1} \frac{\sqrt{v(\vartheta, h)}}{u(\vartheta)} d \vartheta, \\
\left(1 / c<h<\frac{a+c+2}{a c-1}\right),
\end{array}
$$

where $g(\vartheta)=m \cos ^{2} \vartheta+n \sin ^{2} \vartheta, \hat{\vartheta}(h)=\vartheta_{1}(h)(1 / a<h<1 / c)$,

$$
\vartheta_{1,2}(h)=\frac{1}{2} \arccos \left[\frac{(a-c)^{2} \pm \sqrt{(a-c)-(a+c+2)(a+c-2-4 / h)}}{a+c+2}\right] .
$$

From (3.1) - (3.4), we know that $\lambda_{i}(h)(i=1-4)$ are differentiable functions of $h$. From the parametric representations of $\Gamma_{i}^{h}(i=1-4)$, we obtain explicit formulas for $\lambda_{i}(h)$, they can be described by using complete elliptic integrals. Since we only need to understand information of Hopf bifurcations and homoclinic and heteroclinic bifurcations for the bifurcation of limit cycles of $(1.1)_{E}$, we neglect them. We next investigate and calculate the values of bifurcation parameters. 
1. Hopf bifurcations. The values of Hopf bifurcation parameters in the origin $0(0,0)$ and centers $A_{i}^{0}(i=1-4)$ are

$$
\begin{aligned}
b_{H}^{0}= & \lambda_{2}(0)+0(\varepsilon)=\left.2\left(m x^{2}+n y^{2}\right)\right|_{0(0,0)}+0(\varepsilon)=0+0(\varepsilon) \\
b_{H}^{A_{i}^{0}} & =\lambda_{4}\left(\frac{a+c+2}{a c-1}\right)+0(\varepsilon)=\left.2\left(m x^{2}+n y^{2}\right)\right|_{A_{i}^{0}}+0(\varepsilon) \\
& =\frac{2[(1+a) m+(1+c) n]}{a c-1}+0(\varepsilon) .
\end{aligned}
$$

2. Homoclinic and heteroclinic bifurcations. To obtain the limit values of $\lambda_{i}(h)(i=1-4)$, as $h \longrightarrow 1 / a$ and $h \longrightarrow 1 / c$ respectively, it is necessary to calculate the following 17 integrals. Here we give the results of the calculations.

$$
\begin{aligned}
& I_{10}=\int_{0}^{\pi / 2} \frac{d \vartheta}{u(\vartheta)}=I_{11}+I_{12} \\
& I_{11}=\int_{0}^{\pi / 2} \frac{\cos ^{2} \vartheta d \vartheta}{u(\vartheta)}=\pi /[8 c(\sqrt{a c}-1)]^{1 / 2} \stackrel{\text { def }}{=} I_{11}(a, c), \\
& I_{12}=\int_{0}^{\pi / 2} \frac{\sin ^{2} \vartheta d \vartheta}{u(\vartheta)}=\pi /[8 a(\sqrt{a c}-1)]^{1 / 2} \stackrel{\text { def }}{=} I_{11}(c, a) \\
& I_{21}=\int_{0}^{\pi / 2} \frac{\sqrt{v(\vartheta, 1 / a)}}{u(\vartheta)}=\frac{c-a}{4 \sqrt{a} c p}\left[f_{1}(a, c)+\frac{2 p}{\sqrt{4} q-p^{2}}\left(f_{2}(a, c)-\pi\right)\right],
\end{aligned}
$$

where $p=p(a, c)=\sqrt{2}\left(\frac{c q+(a+c+2 a c)}{c}\right)^{1 / 2}, q=q(a, c)=\sqrt{a / c}(a+c+2)$,

$$
\begin{aligned}
& f_{1}(a, c) \stackrel{\text { def }}{=} \ln \frac{2(a+1)-p \sqrt{2(a+1)}+q}{2(a+1)+p \sqrt{2(a+1)}+q}, \\
& f_{2}(a, c) \stackrel{\text { def }}{=} \arctan \frac{2 \sqrt{2(a+1)}-p}{\sqrt{4 q}-p^{2}}+\arctan \frac{2 \sqrt{2(a+1)}+p}{\sqrt{4 q-p^{2}}} \\
& I_{22}=\int_{0}^{\vartheta_{1}(1 / c)} \frac{\sqrt{v(\vartheta, 1 / c)} d \vartheta}{u(\vartheta)}=\frac{a-c}{4 \sqrt{c} a p_{0}}\left[f_{1}(c, a)=\frac{2 p_{0}}{\sqrt{4 q_{0}-p_{0}^{2}}} f_{2}(c, a)\right],
\end{aligned}
$$

where $p_{0}=p(c, a), q_{0}=q(c, a)$.

$$
\begin{gathered}
J_{11}=\int_{0}^{\pi / 2} \frac{\cos ^{2} \vartheta d \vartheta}{u^{2}(\vartheta)}=\frac{\pi\left[\sqrt{a}\left(2-3 a c-2 c^{2}-2 c\right)-\sqrt{c}(2 a c+a+c)\right]}{8 c(1 a-c)[2 a c(\sqrt{a c}-1)]^{1 / 2}} \\
\stackrel{\text { def }}{=} J_{11}(a, c), \\
J_{12}=\int_{0}^{\pi / 2} \frac{\sin ^{2} \vartheta d \vartheta}{u^{2}(\vartheta)}=J_{11}(c, a), J_{10}=\int_{0}^{\pi / 2} \frac{d \vartheta}{u^{2}(\vartheta)}=J_{11}+J_{12},
\end{gathered}
$$




$$
\begin{aligned}
J_{21}=\int_{0}^{\pi / 2} \frac{\sqrt{v(\vartheta, 1 / a)} \cos ^{2} \vartheta d \vartheta}{u^{2}(\vartheta)}=\frac{(c-a)^{2}}{\sqrt{a} c^{2}}\left[f_{01}(a, c)\right. & +\frac{B}{2} f_{1}(a, c) \\
& +\frac{2 D+B p}{\sqrt{4 q-p^{2}}}\left(f_{2}(a, c)-\pi\right]
\end{aligned}
$$

where

$$
\begin{gathered}
A=\left[\left(p^{2}-2 q\right)(3 a+c+4)-4(a+1)(a+c+2)+2 q^{2}-\left(2 q-p^{2}\right)^{2}\right] \\
/\left[2 p^{2}\left(p^{2}-4 q\right)\right] \stackrel{\text { deft }}{=} A(a, c), \quad A_{0}=A(c, a), \quad B_{0}=B(c, a) \\
B=\left[\left(2 q-p^{2}\right)(2 A+1)+(3 a+c+4)+4 q(A+1)\right] / 8 p q \stackrel{\text { def }}{=} B(a, c) \\
D=-\left[\left(2 q-p^{2}\right)(2 A+1)+(3 a+c+4)\right] / 8 q \stackrel{\text { def }}{=} D(a, c), \quad D_{0}=D(c, a) \\
f_{01}(a, c) \stackrel{\text { def }}{=} \frac{\sqrt{2(a+1)\left[\left(2 q-p^{2}\right)(2 A+1)+(3 a+c+4)\right] / 4+A(2 a+2)^{3 / 2}}}{4(a+1)^{2}+2\left(2 q-p^{2}\right)(a+1)+q^{2}} \\
J_{22}=\int_{0}^{\pi / 2} \frac{\sqrt{v(\vartheta, 1 / a)} \sin ^{2} \vartheta d \vartheta}{u^{2}(\vartheta)}=\frac{(c-a)^{2}}{\sqrt{a} c^{2}}\left[f_{02}(a, c)+\frac{F}{2} f_{1}(a, c)\right. \\
\left.+\frac{2 G+F p}{\sqrt{4 q-p^{2}}}\left(f_{2}(a, c)-\pi\right)\right]
\end{gathered}
$$

where

$$
\begin{aligned}
& E=\frac{p^{2}-2 q-2(a+c+2)}{2 p^{2}\left(p^{2}-4 q\right)} \stackrel{\text { def }}{=} E(a, c), \quad E_{0}=E(c, a), \\
& F=\left[2 E\left(2 q-p^{2}\right)+4 q E+1\right] /(8 p q) \stackrel{\text { def }}{=} F(a, c), \quad F_{0}=F(c, a), \\
& G=-\left[2 E\left(2 q-p^{2}\right)+1\right] /(8 q) \stackrel{\text { def }}{=} G(a, c), \quad G_{0}=G(c, a), \\
& f_{02}(a, c) \stackrel{\text { def }}{=} \frac{\sqrt{2(a+1)}\left[2 E\left(2 q-p^{2}\right)+1\right] / 4+E(2 a+2)^{3 / 2}}{4(a+1)^{2}+2\left(2 q-p^{2}\right)(a+1)+q^{2}} \\
& J_{20}=\int_{0}^{\pi / 2} \frac{\sqrt{v(\vartheta, 1 / a)} d \vartheta}{u^{2}(\vartheta)}=J_{21}+J_{22}, \\
& J_{31}=\int_{0}^{\pi / 2} \frac{\frac{v(\vartheta, 1 / a) \cos ^{2} \vartheta d \vartheta}{u^{2}(\vartheta)}=J_{11}-\frac{1}{a} I_{11},}{J_{32}}=\int_{0}^{\pi / 2} \frac{v(\vartheta, 1 / a) \sin ^{2} \vartheta d \vartheta}{u^{2}(\vartheta)}=J_{12}-\frac{1}{a} I_{12}, \quad J_{30}=J_{31}+J_{32}, \\
& J_{41}=\int_{0}^{\vartheta 1(1 / c)} \frac{\sqrt{v(\vartheta, 1 / c)} \cos ^{2} \vartheta d \vartheta}{u^{2}(\vartheta)}=\frac{(a-c)^{2}}{\sqrt{c} a^{2}}\left[\begin{array}{c}
f_{02}(c, a)+\frac{F_{0}}{2} f_{1}(c, a) \\
\left.+\frac{2 G_{0}+F_{0} p_{0}}{\sqrt{4} q_{0}-p_{0}^{2}} f_{2}(c, a)\right]
\end{array}\right]
\end{aligned}
$$




$$
\begin{aligned}
& J_{42}=\int_{0}^{\vartheta_{2}(1 / c)} \frac{\sqrt{v(\vartheta, 1 / c)} \sin ^{2} \vartheta d \vartheta}{u^{2}(\vartheta)}=\frac{(a-c)^{2}}{\sqrt{c a^{2}}}\left[f_{01}(c, a)+\frac{B_{0}}{2} f_{1}(c, a)\right. \\
&\left.+\frac{2 D_{0}+B_{0} p_{0}}{\sqrt{4 q_{0}-p_{0}^{2}}} f_{2}(c, a)\right], \\
& J_{40}=\int_{0}^{\vartheta_{1}(1 / c)} \frac{\sqrt{v(\vartheta, 1 / c)} d \vartheta}{u^{2}(\vartheta}=J_{41}+J_{42} .
\end{aligned}
$$

Using (3.1) - (3.4) and the previous integrals, we have

$$
\begin{aligned}
& \lambda_{1}\left(\frac{1}{a}\right)=\frac{m\left(J_{11}+2 J_{21}+J_{31}\right)+n\left(J_{12}+2 J_{22}+J_{32}\right)}{I_{10}+I_{21}}, \\
& \lambda_{2}\left(\frac{1}{a}\right)=\frac{m\left(J_{11}-2 J_{21}+J_{31}\right)+n\left(J_{12}+2 J_{22}+J_{32}\right)}{I_{10}-I_{21}}, \\
& \lambda_{3}\left(\frac{1}{a}\right)=\frac{2\left(m J_{21}+n J_{22}\right)}{I_{21}}, \\
& \lambda_{3}\left(\frac{1}{c}\right)=\lambda_{4}\left(\frac{1}{c}\right)=\frac{2\left(m J_{41}+n J_{42}\right)}{I_{22}} .
\end{aligned}
$$

Note that $\lambda_{i}\left(\frac{1}{a}\right)$ and $\lambda_{j}\left(\frac{1}{c}\right)(i=1-3, j=3,4)$ give respectively the parameter values of heteroclinic and homoclinic bifurcations.

Proposition 3.1.

$$
\begin{aligned}
& \text { If } \lambda_{1}\left(\frac{1}{a}\right)>\lambda_{2}\left(\frac{1}{a}\right), \text { than } \lambda_{3}\left(\frac{1}{a}\right)>\lambda_{1}\left(\frac{1}{a}\right)>\lambda_{2}\left(\frac{1}{a}\right) ; \\
& \text { If } \lambda_{1}\left(\frac{1}{a}\right)<\lambda_{2}\left(\frac{1}{a}\right) \text {, than } \lambda_{3}\left(\frac{1}{a}\right)<\lambda_{1}\left(\frac{1}{a}\right)<\lambda_{2}\left(\frac{1}{a}\right) .
\end{aligned}
$$

Proof: From (3.1) - (3.4), we know that

$$
\lambda_{1}\left(\frac{1}{a}\right)-\lambda_{2}\left(\frac{1}{a}\right)=\frac{\psi_{1}(1 / a) \phi_{2}(1 / a)-\psi_{2}(1 / a) \phi_{1}(1 / a)}{\phi_{1}(1 / a) \phi_{2}(1 / a)},
$$

and

$$
\lambda_{3}\left(\frac{1}{a}\right)-\lambda_{2}\left(\frac{1}{a}\right)=\frac{\phi_{1}(1 / a) \phi_{2}(1 / a)-\psi_{2}(1 / a) \phi_{1}(1 / a)}{\phi_{i}(1 / a)\left[\phi_{1}(1 / a)-\phi_{2}(1 / a)\right]}, i=1,2
$$

It is easy to see that

$$
\phi_{3}\left(\frac{1}{a}\right)=\phi_{1}\left(\frac{1}{a}\right)-\phi_{2}\left(\frac{1}{a}\right)>0, \quad \phi_{i}\left(\frac{1}{a}\right)>0 \quad(i=1,2) .
$$

Thus, (3.11) and (3.12) gives the conclusion. 
3. The values of saddle points $S_{j}$ - the detection values of direction of homoclinic and heteroclinic bifurcations. Under the condition that the unperturbed vector field has some symmetries, the sign of the values of a saddle point can be used to determine the stability of a bifurcating closed orbit from a homoclinic or heteroclinic loop, and give the signs of $\lambda_{i}^{\prime}(1 / a), \lambda_{j}^{\prime}(1 / c)(i=1,2,3, j=3,4)$.

At the saddle points $S_{1}$ and $S_{2}$, when the parameter $\lambda$ takes the values of $\lambda_{j}(1 / c)(j=3,4)$, we have

$$
\begin{aligned}
\sigma_{3,4} & =2 \varepsilon\left[\left.2\left(m x^{2}+n y^{2}\right)\right|_{s_{1}}-\lambda_{3}(1 / c)\right]+0\left(\varepsilon^{2}\right) \\
& =4 \varepsilon\left[\frac{m\left(I_{22}-c J_{41}\right)-n c J_{42}}{c I_{22}}\right]+0\left(\varepsilon^{2}\right) .
\end{aligned}
$$

Similarly, at the saddle points $S_{3}$ and $S_{4}$, when $\lambda=\lambda_{i}(1 / a)(i=1,2,3)$, we have

$$
\sigma_{i}=2 \varepsilon\left[\left.2\left(m x^{2}+n y^{2}\right)\right|_{S_{3}}-\lambda_{i}(1 / a)\right]+O\left(\varepsilon^{2}\right) .
$$

By using (3.7) - (3.9), we obtain

$$
\sigma_{1}=2 \varepsilon \frac{\left[2\left(I_{10}+I_{21}\right)-a\left(J_{12}+2 J_{22}+J_{32}\right)\right] n-m a\left(J_{11}+2 J_{21}+J_{31}\right)}{a\left(I_{10}+I_{21}\right)}+0\left(\varepsilon^{2}\right)
$$

$$
\sigma_{2}=2 \varepsilon \frac{\left[2\left(I_{10}-I_{21}\right)-a\left(J_{12}-2 J_{22}+J_{32}\right)\right] n-m a\left(J_{11}-2 J_{21}+J_{31}\right)}{a\left(I_{10}-I_{21}\right)}+0\left(\varepsilon^{2}\right)
$$

$$
\sigma_{3}=4 \varepsilon \frac{\left(I_{21}-a J_{22}\right) n-a m J_{21}}{a I_{21}}+0\left(\varepsilon^{2}\right)
$$

\section{Global and local bifurcations in the cases $m=n$}

If we let $m=n$, then (1.1) becomes

$$
\begin{aligned}
& \frac{d x}{d t}=y\left(1+x^{2}-a y^{2}\right)+\varepsilon x\left(m x^{2}+m y^{2}-\lambda\right), \\
& \frac{d y}{d t}=-x\left(1-c x^{2}+y^{2}\right)+\varepsilon y\left(m x^{2}+m y^{2}-\lambda\right) .
\end{aligned}
$$

Hence, from 3 , we have

$$
\begin{aligned}
\lambda_{1}\left(\frac{1}{a}\right) & =\frac{m\left(J_{10}+2 J_{20}+J_{30}\right)}{I_{10}+I_{21}}, \lambda_{2}\left(\frac{I}{a}\right)=\frac{m\left(J_{10}-2 J_{20}+J_{30}\right)}{I_{10}-I_{21}}, \\
\lambda_{3}\left(\frac{I}{a}\right) & =\frac{2 m J_{20}}{I_{21}}, \quad \lambda_{4}\left(\frac{I}{c}\right)=\frac{2 m J_{40}}{I_{22}}, \\
b_{H}^{A_{i}^{0}} & =\frac{2 m(a+c+2)}{a c-1}+0(\varepsilon), b_{H}^{0}=0+0(\varepsilon) .
\end{aligned}
$$


Lemma 4.1. $\lambda_{3}^{\prime}(h)>0, \lambda_{4}^{\prime}(h)>0$ and $\lim _{h \rightarrow 1 / a+0} \lambda_{3}^{\prime}(h)=+\infty$,

$$
\lim _{h \rightarrow 1 / c-0} \lambda_{3}^{\prime}(h)=\lim _{h \rightarrow 1 / c+0} \lambda_{4}^{\prime}(h)=+\infty
$$

Proof: Since

$$
\lambda_{i}^{\prime}(h)=\left[\psi_{i}^{\prime} \phi_{i}-\phi_{i}^{\prime} \psi_{i}\right] / \phi_{i}^{2}, \quad(i=3,4),
$$

we see from (3.4) that

$$
\begin{aligned}
& I \stackrel{\text { def }}{=} \psi_{3}^{\prime} \phi_{3}-\phi_{3}^{\prime} \psi_{3} \\
& \quad=\frac{-1}{2}\left[\int_{-\vartheta_{1}(h)}^{\vartheta_{1}(h)} \frac{d \vartheta}{u \sqrt{v}} \int_{-\vartheta_{1}(h)}^{\vartheta_{1}(h)} \frac{\sqrt{v}}{u} d \vartheta-\int_{-\vartheta_{1}(h)}^{\vartheta_{1}(h)} \frac{d \vartheta}{\sqrt{v}} \int_{-\vartheta_{1}(h)}^{\vartheta_{1}(h)} \frac{\sqrt{v} d \vartheta}{u^{2}}\right] .
\end{aligned}
$$

This integral formula can be written as a double integral [4]:

$$
I \stackrel{\text { def }}{=} \int_{-\vartheta_{1}(h)}^{\vartheta_{1}(h)} \int_{-\vartheta_{1}(h)}^{\vartheta_{1}(h)} \frac{\left[u_{1}\left(\hat{\vartheta}_{1}\right)-u_{2}\left(\hat{\vartheta}_{2}\right)\right]}{u_{1}^{2}\left(\hat{\vartheta}_{1}\right) u_{2}^{2}\left(\hat{\vartheta}_{2}\right) \sqrt{v_{1} v_{2}}} d \hat{\vartheta}_{1} d \hat{\vartheta}_{2}>0 .
$$

When $h$ tends to $1 / a$ or $1 / c$, at $\vartheta=0$ of $\vartheta=\pi / 2$, the function $[v(\vartheta, h)]^{-1 / 2}$ is unbounded. From (4.2), this implies that

$$
\lim _{h \rightarrow 1 / \alpha+0} \lambda_{3}^{\prime}(h)=\lim _{h \rightarrow 1 / c-0} \lambda_{3}^{\prime}(h)=+\infty
$$

Similarly, we can have the result for $\lambda_{4}^{\prime}(h)$. Lemma 4.1 implies that $\sigma_{3,4}<$ $0, \sigma_{3}<0$.

Lemma 4.2. $\frac{d}{d h}\left(\frac{\psi_{1}^{\prime}(h)}{\phi_{1}^{\prime}(h)}\right)<0, \frac{d}{d h}\left(\frac{\psi_{2}^{\prime}(h)}{\phi_{2}^{\prime}(h)}\right)<0$.

Proof: For $i=1,2$, we have

$$
\frac{d}{d h}\left(\frac{\psi_{i}^{\prime}(h)}{\phi_{2}^{\prime}(h)}\right)=\frac{1}{\left[\phi_{i}^{\prime}(h)\right]^{2}}\left[\psi_{i}^{\prime \prime}(h) \phi_{i}^{\prime}(h) \psi_{i}^{\prime}(h)\right]
$$

Using (3.1) and (3.2), we know that

$$
\begin{aligned}
J & \stackrel{\text { def }}{=} \psi_{i}^{\prime \prime}(h) \phi_{i}^{\prime}(h)-\phi_{i}^{\prime \prime}(h) \psi_{i}^{\prime}(h) \\
& =\mp \frac{1}{4}\left[\int_{0}^{\pi / 2} \frac{d \vartheta}{\sqrt{v}} \int_{0}^{\pi / 2} \frac{d \vartheta}{v^{3 / 2}}-\int_{0}^{\pi / 2} \frac{d \vartheta}{u \sqrt{v}} \int_{0}^{\pi / 2} \frac{u d \vartheta}{v^{3 / 2}}+\int_{0}^{\pi / 2} \frac{d \vartheta}{u} \int_{0}^{\pi / 2} \frac{u d \vartheta}{v^{3 / 2}}\right]
\end{aligned}
$$

$$
=\mp\left[\int_{0}^{\pi / 2} \int_{0}^{\pi / 2} \frac{1}{\left(v_{1} v_{2}\right)^{3 / 2}}\left(\frac{v_{1}^{3 / 2}+v_{1} v_{2}}{1+\sqrt{v_{1}}}+\frac{v_{2}^{3 / 2}+v_{1} v_{2}}{1+\sqrt{v_{2}}}\right) d \hat{\vartheta}_{1} d \hat{\vartheta}_{2}\right] \text {. }
$$

The signs - and + on the right hand of (4.3) are respectively corresponding to the cases of $i=1$ and $i=2$. Thus, (4.3) gives the conclusion of lemma 4.2. 
Lemma 4.3. [5] For $h \in\left(0, h_{0}\right)$, assume that the functions $\phi(h)$ and $\psi(h)$ are sufficiently smooth, nonnegative and monotone increasing, the function $\psi^{\prime}(h) / \phi^{\prime}(h)$ is nonnegative and monotone increasing (decreasing). Then the function $\psi(h) / \phi(h)$ must satisfy one of the following properties:

(i) monotone increasing after it decreases to a minimum (monotone decreasing after it increases to a maximum);

(ii) monotone increasing;

(iii) monotone decreasing.

In particular, if $\phi(0)=\psi(0)=0$ and $\lim _{h \rightarrow 0} \psi(h) / \phi(h)=a \geq 0$ (or $<0$ ), then $\psi(h) / \phi(h)$ must be increasing (decreasing).

Lemma 4.4. $\lim _{h \rightarrow-\infty} \lambda_{1}(h)=+\infty$.

Proof: Write $r_{m}^{2}=\min _{\vartheta \in\{0,2 \pi\}} r_{+}^{2}(\vartheta, h), h \in(-\infty, 0)$, because $\Gamma_{ \pm}^{h}$ extends infinitely as $h \longrightarrow-\infty$, it follows that $r^{2} \longrightarrow+\infty$, as $h \longrightarrow-\infty$. We have

$$
\lim _{h \longrightarrow-\infty} \lambda_{1}(h) \geq \lim _{h \longrightarrow-\infty}\left(r_{m}^{2} \int_{0}^{\pi / 2} r_{+}^{2} d \vartheta / \int_{0}^{\pi / 2} r_{+}^{2} d \vartheta\right)=\lim _{h \longrightarrow-\infty} r_{m}^{2}=+\infty
$$

Lemma 4.5. If $\lambda_{1}\left(\frac{1}{a}\right)>\frac{2}{a}$, then $\lambda_{1}(h)$ will be monotone increasing after it decreases to a minimum; if $\lambda_{1}\left(\frac{1}{a}\right)<\frac{2}{a}$, then $\lambda_{1}(h)$ is monotone decreasing, and $\lambda_{2}(h)$ is monotone increasing.

Proof: If $\lambda_{1}\left(\frac{1}{a}\right)>\frac{2}{a}$, then $\sigma_{1}<0$, i.e., $\lim _{h \rightarrow 1 / a-0} \lambda_{1}(1 / a)=+\infty$. Thus, Lemma $4.2-4.4$ can follow the first part of the conclusion of Lemma 4.5. On the other hand, $\phi_{2}(0)=\psi_{2}(0)=0$, Lemma 4.2 and Lemma 4.3 lead to the result stated for $\lambda_{2}^{\prime}(h)$

Lemma 4.6. For the system $(4.1)_{\varepsilon}$, the inequality $\lambda_{3}\left(\frac{1}{a}\right)>\lambda_{1}\left(\frac{1}{a}\right)>\lambda_{2}\left(\frac{1}{a}\right)$ holds.

Proof: We have

$$
\lambda_{1}\left(\frac{1}{a}\right)-\lambda_{2}\left(\frac{I}{a}\right)=\frac{2\left(J_{10}+J_{30}\right) J_{11}+4 J_{20} J_{10}}{I_{20}^{2}-I_{11}^{2}}>0 .
$$

by using Proposition 3.1, we have Lemma 4.6

These lemmas enable us to determine two types of detection curves of $(4.3)_{\epsilon}$, shown as Fig. 4.1 (a) (b). 


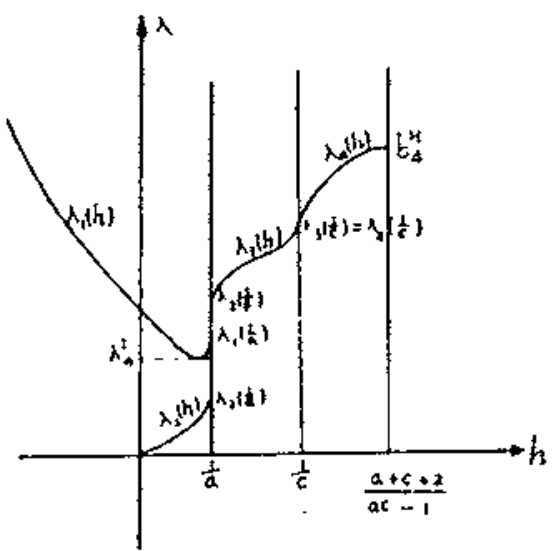

(a)

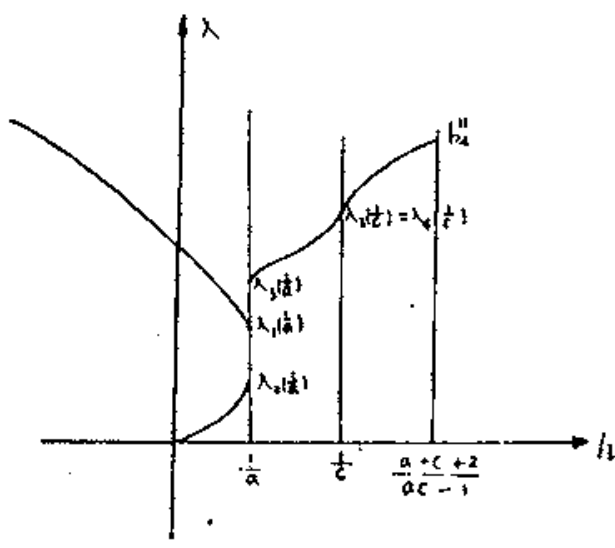

(b)

If $\lambda_{1}\left(\frac{1}{a}\right)<\frac{2}{a}$

Fig. 4.I The detection curves of $(1.1)_{\varepsilon}$ when $m=n$.

\section{Bifurcation set in the $(n, m)$ plane}

We now consider the general case of $m \neq n$ for the system $(1.1)_{\epsilon}$.

Lemma 5.1. $\lim _{h \rightarrow-\infty} \lambda_{1}(h)=+\infty$, if $m+k_{1}(\dot{a}, c) n>0 ; \lim _{h \rightarrow-\infty} \lambda_{1}(h)=$ $-\infty$, if $m+k_{1}(a, c) n<0$, where $k_{1}(a, c)=I_{12} / I_{1}$.

Proof: From (3.1), we have

$$
\begin{aligned}
\psi_{1}(h) & =\int_{0}^{\pi / 2}\left[\frac{1+\sqrt{v(\vartheta, h)}}{u(\vartheta)}\right]^{2} g(\vartheta) d \vartheta \\
& =2 \int_{0}^{\pi / 2} \frac{g(\vartheta)}{u^{2}(\vartheta)} d \vartheta+2 \int_{0}^{\pi / 2} \frac{\sqrt{v(\vartheta, h)}}{u^{2}(\vartheta)} g(\vartheta) d \vartheta-h \int_{0}^{\pi / 2} \frac{g(\vartheta)}{u(\vartheta)} d \vartheta \\
& =(\ldots)+(\ldots)-h I_{11}\left(m+k_{1}(a, c) n\right) .
\end{aligned}
$$

Since $\phi_{1}(h)$ is exactly a quarter of the area insider $\Gamma_{1}^{h}$, we have $\phi_{1}(h) \rightarrow+\infty$ as $h \longrightarrow+\infty$. Clearly, as $h \longrightarrow-\infty$, the sign of $\lim \lambda_{1}(h)$ is determined by the third term of (5.1). Hence, Lemma 5.1 is true. 
Next, we apply the results of 3 to partition the parameter plane $(n, m)$ as regions of angles, inside which there are different detection curves of $(1.1)_{\varepsilon}$. Without loss of the generality, we only discuss the case of $m \geq 0$. In the upper half plane of $(n, m)$, we have 18 half straight lines with linear equations as follows.

1. The line $l_{1}$ is use to distinguish the sign of $\lim _{h \rightarrow-\infty} \lambda_{\mathrm{I}}(h)$.

$$
l_{1}: m=-k_{1}(a, c) n, \quad k_{1}(a, c)=I_{12} / I_{11} .
$$

2. Straight lines $l_{2}-l_{5}$ show that values of saddle points of $(1.1)_{\varepsilon}$ are equal to zero.

$$
l_{2}: \sigma_{1}=0, m=\left[\frac{2\left(I_{10}+I_{21}\right)-a\left(J_{12}+2 J_{22}+J_{32}\right)}{a\left(J_{11}+2 J_{21}+J_{31}\right)}\right] n=k_{2}(a, c) n ;
$$

$$
l_{3}: \sigma_{2}=0, m=\left[\frac{2\left(I_{10}-I_{21}\right)-a\left(J_{12}-2 J_{22}+J_{32}\right)}{a\left(J_{11}+2 J_{21}+J_{31}\right)}\right] n=k_{3}(a, c) n ;
$$

$$
l_{4}: \sigma_{3}=0, m=\left[\frac{I_{21}-a J_{22}}{a J_{21}}\right] n=k_{4}(a, c) n ;
$$

$$
l_{5}: \sigma_{3,4}=0, m=\left[\frac{c J_{42}}{I_{22}-c J_{41}}\right] n=k_{\mathrm{S}}(a, c) n .
$$

3. Straight lines $l_{6}-l_{13}$ determine the relations between Hopf bifurcation parameters and homoclinic, heteroclinic bifurcation parameters.

(i) $\lambda_{1}(1 / a)=0$,

$$
\iota_{6}: m=-\left[\frac{J_{12}+2 J_{22}+J_{32}}{J_{11}+2 J_{21}+J_{31}}\right] n=k_{6}(a, c) n ;
$$

(ii) $\lambda_{2}(I / a)=0$,

$$
l_{7}: m=-\left[\frac{J_{12}-2 J_{22}+J_{32}}{J_{11}-2 J_{21}+J_{31}}\right] n=k_{7}(a, c) n ;
$$

(iii) $\lambda_{3}(1 / a)=0$,

$$
l_{8}: m=-\left(\frac{J_{22}}{J_{21}}\right) n=k_{8}(a, c) n
$$


(iv) $\lambda_{1}(l / c)=0$

$$
l_{9}: m=-\left(\frac{J_{42}}{J_{41}}\right) n=k_{9}(a, c) n
$$

(v) $b_{H}^{A_{i}^{0}}-\lambda_{1}(1 / a)=0$,

$l_{10}: m=-\left[\frac{2(1+c)\left(I_{10}+I_{21}\right)-(a c-1)\left(J_{12}+2 J_{22}+J_{32}\right)}{2(1+a)\left(I_{10}+I_{21}\right)-(a c-1)\left(J_{11}+2 J_{21}+J_{31}\right)}\right] n=k_{10}(a, c) n$;

(vi) $b_{h}^{A_{i}^{0}}-\lambda_{2}(1 / a)=0$,

$\iota_{11}: m=-\left[\frac{2(1+c)\left(I_{10}-I_{21}\right)-(a c-1)\left(J_{12}-2 J_{22}+J_{32}\right)}{2(1+a)\left(I_{10}-I_{21}\right)-(a c-1)\left(J_{11}-2 J_{21}+J_{31}\right)}\right] n=k_{11}(a, c) n$;

(vii) $b_{H}^{A_{i}^{0}}-\lambda_{3}(l / a)=0$

$$
l_{12}: m=-\left[\frac{(1+c) \Gamma_{21}-(a c-1) J_{22}}{(1+a) I_{21}-(a c-1) J_{21}}\right] n=k_{12}(a, c) n
$$

(viii) $b_{H}^{A_{i}^{0}}-\lambda_{4}(1 / a)=0$

$$
l_{13}: m=-\left[\frac{(1+c) I_{22}-(a c-1) J_{42}}{(1+a) I_{22}-(a c-1) J_{41}}\right]=k_{13}(a, c) n
$$

4. Straight lines $l_{14}-l_{17}$ determine the relations of homoclinic bifurcation parameters and heteroclinic bifurcation parameters.

(i) $\lambda_{1}(1 / a)-\lambda_{3}(1 / c)=0$,

$$
l_{14}: m=-\left[\frac{I_{21}\left(J_{12}+J_{32}\right)-2 I_{10} J_{22}}{I_{21}\left(J_{11}+J_{31}\right)-2 I_{10} J_{21}}\right]=k_{14}(a, c) n
$$

(ii) $\lambda_{1}(1 / a)-\lambda_{4}(1 / c)=0$,

(5.16) $l_{15}: m=-\left[\frac{I_{22}\left(J_{12}+2 J_{22}+J_{32}\right)-2\left(I_{10}+I_{21}\right) J_{42}}{I_{22}\left(J_{11}+2 J_{21}+J_{31}\right)-2\left(I_{10}+I_{21}\right) J_{41}}\right] n=k_{15}(a, c) n_{\text {i }}$

(iii) $\lambda_{2}(1 / a)-\lambda_{4}(1 / c)=0$,

$$
l_{16}: m=-\left[\frac{I_{22}\left(J_{12}-2 J_{22}+J_{32}\right)-2\left(I_{10}-I_{21}\right) J_{42}}{I_{22}\left(J_{11}-2 J_{21}+J_{31}\right)-2\left(I_{10}-I_{21}\right) J_{41}}\right] n=k_{16}(a, c) n_{i}
$$


(iv) $\lambda_{3}(1 / a)-\lambda_{4}(1 / c)=0$

$$
l_{17}: m=-\left[\frac{I_{22} J_{22}-I_{21} J_{42}}{I_{22} J_{21}-I_{22} J_{41}}\right] n=k_{17}(a, c) n .
$$

5. The straight line $l_{18}$ determines the relation between Hopf bifurcation parameters, i.e. $b_{H}^{A_{i}^{0}}-b_{H}^{0}=0$.

$$
l_{18}: m=-\left(\frac{1+c}{1+a}\right) n=k_{28}(a, c) n .
$$

It is easy to see that the previous 18 straight lines together with the addition of the $n$-axis, partition the half-parameter plane $(n, m)$ as 19 angle regions. Using the integral formulas in 3 to compute $k_{i}(a, c),(i=1-18)$, and letting $k_{i}(a, c)=\operatorname{tg} \vartheta_{i}(a, c)$, we obtain:

Lemma 5.2. There exist many parameter pairs $(a, c)$ with $a>c>0$ and ac $>1$, such that $0<\vartheta_{3}<\vartheta_{5}<\vartheta_{7}<\vartheta_{13}<\vartheta_{1}<\vartheta_{12}<\vartheta_{10}<\vartheta_{18}<\vartheta_{6}<$ $\vartheta_{8}<\vartheta_{11}<\vartheta_{9}<\vartheta_{14}<\vartheta_{16}<\vartheta_{15}<\vartheta_{17}<\vartheta_{1}<\vartheta_{2}<\pi$.

As an example, take $a=6, c=2$, we have $k_{1}=-0.577, k_{2}=-0.01829, k_{3}=$ $6.5749, k_{4}=-0.054, k_{5}=-3.41, k_{6}=-0.4117, k_{7}=-1.85, k_{8}=-0.405$, $k_{9}=-0.3744, k_{10}=-0.462, k_{11}=-0.39, k_{12}=-0.496, k_{13}=-0.819, k_{14}=$ $-0.3526, k_{15}=-0.26, k_{16}=-0.328, k_{17}=-0.206, k_{18}=-0.43$.

In $(n, m)$ half plane, we compute all 18 half straight lines to get the partition of the parameter plane shown as Fig. 5.1. Corresponding to every angle region $R_{i}(i=1-19)$ of $(n, m)$ half plane, the sketches of the detection curves have been drawn in the table 5.1 .

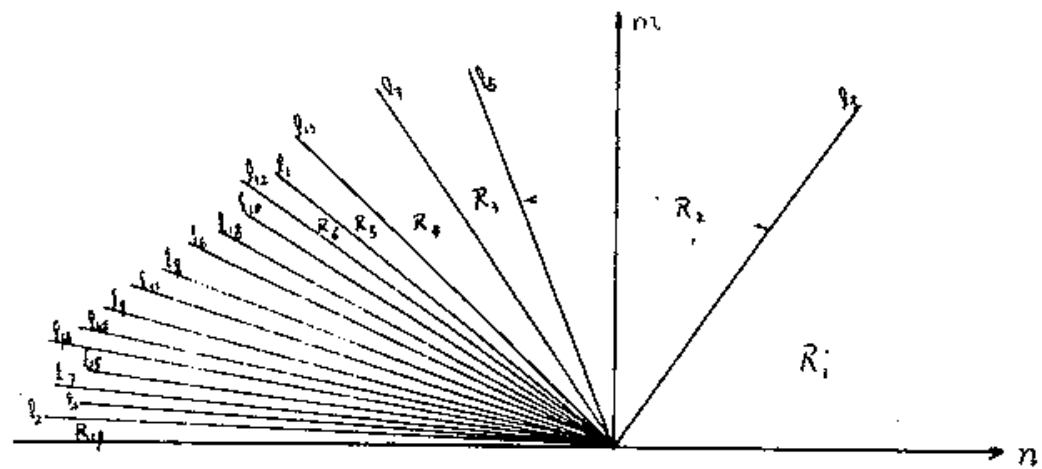

Fig. 5.1 The partition of $(n, m)$ half plane of parameters. 


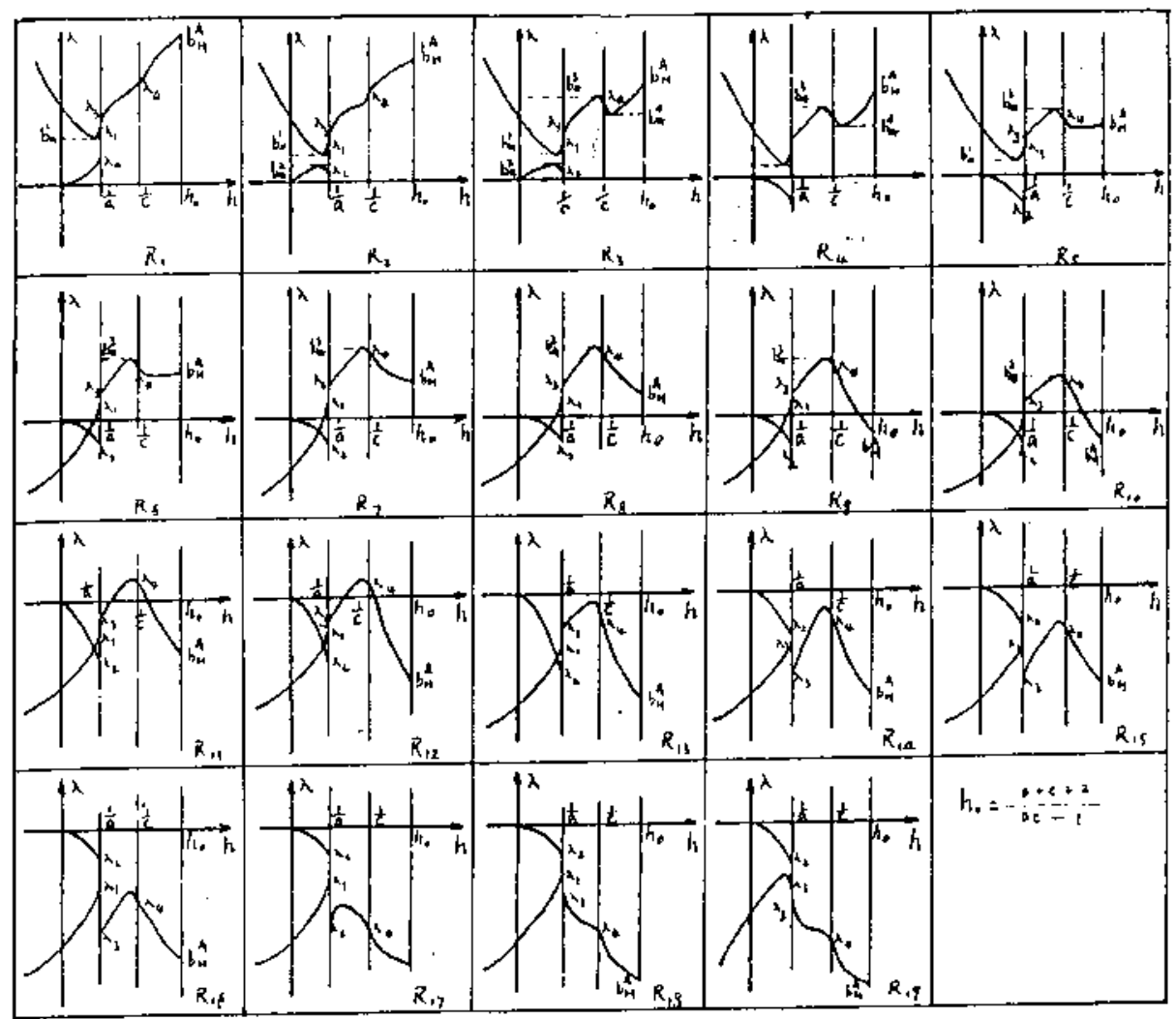

Table 5.1 The sketches of all detection curves of $(1.1)_{\mathrm{F}}$.

In order to understand the phenomenon of bifurcation of limit cycles, we give a group of phase portraits when the points $(n, m)$ are inside the angle region $R_{3}$. They are shown as Fig. 5.2. Using table 5.1, we see that there exist many interesting distributions of limit cycles and homoclinic or heteroclinic loops, for the sake of brevity, we omit them.

Let $C_{m}^{k}$ denote a nest of $k$ limit cycles which encloses $m$ singular points. The sign $\subset$ is used to shown enclosing relations between limit cycles. And the sign + is used to divide limit cycles enclosing different critical points. Denote simply that $C_{m}^{k}+C_{m}^{k}=2 C_{m}^{k}$, etc.

On the basis of the invariance of vector field of (1.1) under a rotation over $\pi$, by the property of detection curves and theorems of Ref. [5], we have the following two theorems 
Theorem 5.1. For any fixed $\varepsilon, 0<\varepsilon<<1$, when $(n, m)$ is inside the angle region $R_{3}$ of Fig. 5.1, as $\lambda$ varies, (1.1) $)_{\epsilon}$ has distributions of limit cycles and homoclinic or heteroclinic loops as follows: $C_{9}^{1}$.

(i) If $b_{H}^{A}<\lambda<+\infty,(1.1)_{\varepsilon}$ has one unstable limit cycle with the distribution

(ii) If $b_{*}^{3}<\lambda<b_{I}^{A},(1.1)_{\varepsilon}$ has slimit cycles with the distribution of $C_{9}^{1} \supset 4 C_{1}^{1}$. $\left.2 C_{1}^{2}\right]$.

(iii) If $\lambda=b_{*}^{3},(1.1)_{\varepsilon}$ has 7 limit cycles with the distribution of $C_{9}^{1} \supset 2\left[C_{3}^{1} \supset\right.$

(iv) If $\lambda_{4}(1 / c)<\lambda<b_{*}^{3},(a . a)_{\varepsilon}$ has 9 limit cycles with the distribution of $C_{9}^{1} \supset 2\left[C_{3}^{2} \supset 2 C_{1}^{1}\right]$.

(v) If $\lambda=\lambda_{4}(1 / c),(1.1)_{\varepsilon}$ has 4 homoclinic loops connecting respectively the points $S_{1}$ and $S_{2}$, with the addition of 7 limit cycles.

(vi) If $b_{*}^{4}<\lambda<\lambda_{4}(1 / c),(1.1)_{\epsilon}$ has 11 limit cycles with the distribution of $C_{9}^{1} \supset 2\left[C_{3}^{1} \supset 2 C_{2}^{2}\right]$. $\left.2 C_{1}^{1}\right]$.

(vii) If $\lambda=b_{*}^{4},(1.1)_{\varepsilon}$ has 7 limit cycles with the distribution of $C_{9}^{1} \supset 2\left[C_{3}^{1} \supset\right.$

(viii) If $\lambda_{3}(1 / a)<\lambda<b_{*}^{4},(1.1)_{\varepsilon}$ has 3 limit cycles with the distribution of $C_{9}^{1} \supset 2 C_{3}^{1}$.

(ix) If $\lambda=\lambda_{3}(1 / a),(1.1)_{\varepsilon}$ has 2 homoclinic loops connecting respectively the points $S_{3}$ and $S_{4}$, with the addition of one limit cycle $C_{9}^{1}$.

(x) If $\lambda=\lambda_{1}(1 / a)$, (1.1) $\varepsilon$ has two heteroclinic loops which are surrounding respectively 9 critical points; outside these loops, there is one limit cycle $C_{9}^{1}$.

By using Table 5.1, we also see that the following result is true.

Theorem 5.2. For a fixed $\varepsilon, 0<\varepsilon<<1$, we have

(i) If $(n, m)$ is inside the angle region $R_{12}$, then the distributions of limit cycles of $(1.1)_{\epsilon}$ are

(a) 7 limit cycles with $2\left[C_{3}^{1} \supset 2 C_{1}^{1}\right]+C_{1}^{3}$ distribution, when $\lambda_{3}(1 / a)<\lambda<0$;

(b) 6 limit cycles with $C_{9}^{1} \supset 5 C_{1}^{1}$ distribution, when $\lambda_{2}(1 / a)<\lambda<\lambda_{1}(1 / a)$;

(c) 4 limits cycles with $2 C_{3}^{2}$ distribution, when $\lambda_{4}(1 / c)<\lambda<b_{*}^{3}$.

(ii) If $(n, m)$ is inside the angle region $R_{10}$, then there are 5 limit cycles of (1.1 $)_{\varepsilon}$ with the distribution $5 C_{1}^{1}$, when $\lambda_{1}(1 / a)<\lambda<0$. This is the Il'jasenko distribution. 


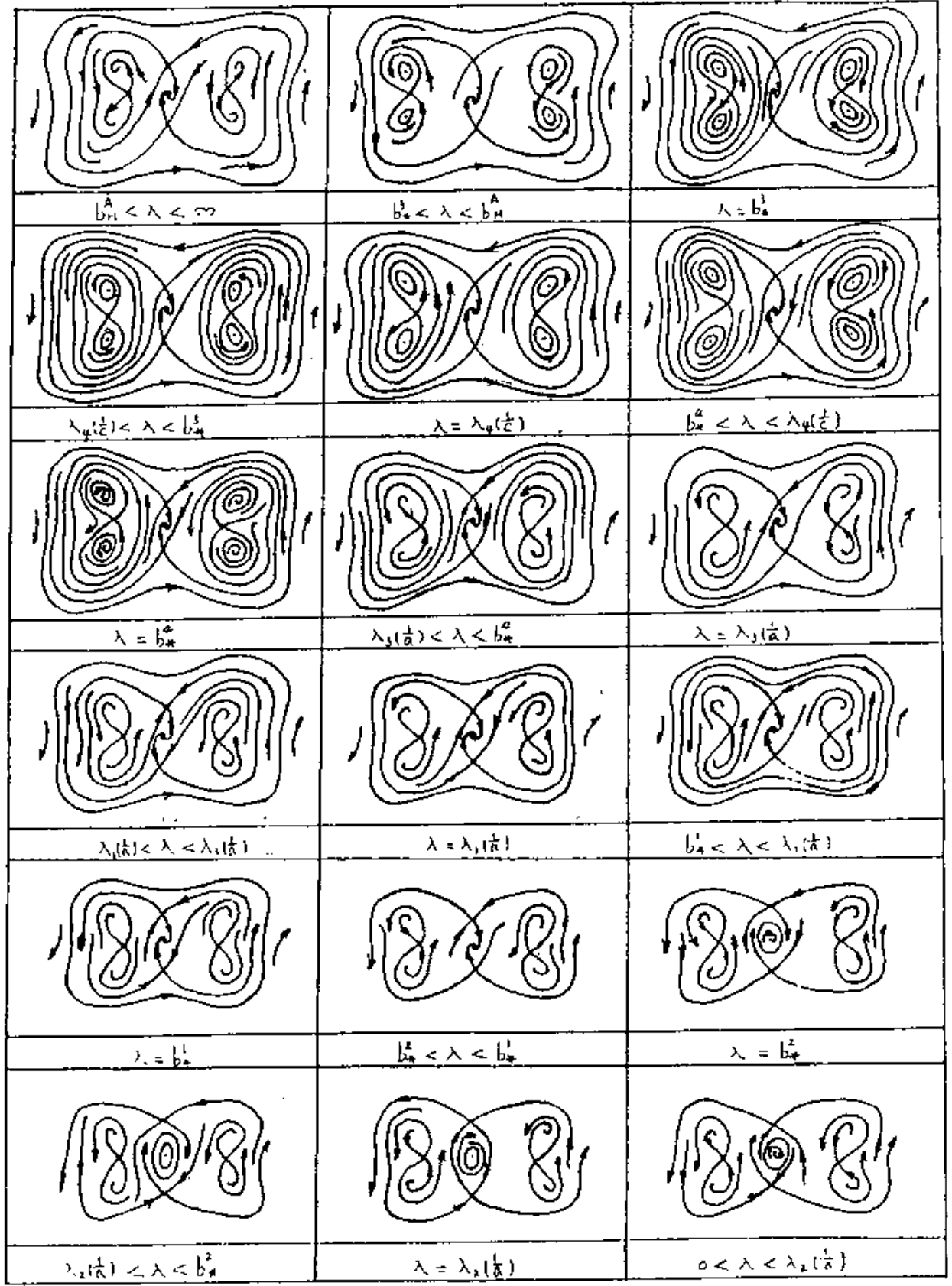

Fig. 5.2 Bifurcations of phase portraits of $(1.1)_{\varepsilon}$ when $(n, m)$ lies inside the angle region $R_{3}$. 


\section{References}

1. V.I. ARNOLD, "Geometric methods in Theory of ordinary differential equations," Springer-Verlag, New York, 1983.

2. LI JIBIN, Researches on the weakened Hilbert's 16th problem, Journal of Kunming Institute of Technology 13 (1988), 94-109.

3. LI JiBIN ETC., Bifurcations of limit cycles forming compound eyes in the cubic system, Chin. Ann. of Math. 8B (1987), 391-403.

4. A.1. NEISTADT, Bifurcations of phase portraits for some equations of stability loss problem at resonance, Appl. Math. and Mech. 42 (1978), 830-840, (in Russian).

5. Li JibIN ETC., Planar cubic Hamiltonian systems and distributions of limit cycles of ( $\left.E_{3}\right)$, Acta Math. Sinica 28 (1985), 509-521.

6. Li JiBIN ETC., Global bifurcations and chaotic behaviour in a disturbed quadratic system with two centers, Acta Math. Appl. Sinica 11 (1988), 312-323.

7. Ju. S. IL'JASENKO, The origin of limit cycles under perturbation of the equation $d w / d z=-R_{z} / R_{u}$, where $R(z, w)$ is a polynomial, Math. Sbornik 78 (1969), 360-373.

8. J. CARR, S.N. ChOW AND J.K. Hale, Abelian integrals and bifurcation theory, Journal of Differential Equations 59 (1985), 413-437.

Jibin Li: Center for Dynamical Systems and Nonlinear Studies

School of Mathematics

Georgia Institutc of Technology

Atlanta, GA 30332

U.S.A.

Zhenrong Liu: Department of Mathematics

Yunnan University

650091 Yunnan

P.R. CHINA

Rebut el 21 de Desembre de 1990 